\title{
Accuracy of the time-dependent Hartree-Fock approximation for uncorrelated initial states
}

\author{
Claude BARDOS*, François GOLSE $\dagger^{\dagger}$ Alex D. GOTTLIEB ${ }^{\ddagger}$ \\ and Norbert J. MAUSER ${ }^{\S}$
}

\begin{abstract}
This article concerns the time-dependent Hartree-Fock (TDHF) approximation of single-particle dynamics in systems of interacting fermions. We find that the TDHF approximation is accurate when there are sufficiently many particles and the initial manyparticle state is any Gibbs equilibrium state for noninteracting fermions (with Slater determinants as a special example). Assuming a bounded two-particle interaction, we obtain a bound on the error of the TDHF approximation, valid for short times. We further show that the error of the TDHF approximation vanishes at all times in the mean field limit.
\end{abstract}

\section{Introduction}

The time-dependent Hartree-Fock (TDHF) equation is a nonlinear Schrödinger equation designed to approximate the evolution of an $n$-electron system. The TDHF equation was first written down by Dirac, both as a system of $n$ coupled Schrödinger equations for occupied orbitals, and as an integro-differential equation for the "density matrix," i.e., the integral kernel

*Univ. Paris 7 \& Lab. J.-L. Lions, Boîte courrier 187, 75252 Paris cedex 05, France (bardos@math.jussieu.fr).

${ }^{\dagger}$ Univ. Paris 7 \& Lab. J.-L. Lions, Boîte courrier 187, 75252 Paris cedex 05, France (golse@math.jussieu.fr).

${ }^{\ddagger}$ Wolfgang Pauli Inst. c/o Inst. f. Mathematik, Univ. Wien, Strudlhofg. 4, A-1090 Wien, Austria (alex@alexgottlieb.com).

${ }^{\S}$ Wolfgang Pauli Inst. c/o Inst. f. Mathematik, Univ. Wien, Strudlhofg. 4, A-1090 Wien, Austria (mauser@courant.nyu.edu). 
$F(x, y, t)$ of the single-particle density operator [11, 12]. In the latter form it reads

$$
\begin{aligned}
i \frac{d}{d t} F(x, y, t) & =-\frac{1}{2}\left(\Delta_{x}-\Delta_{y}\right) F(x, y, t)+\left(V_{e x t}(x)-V_{e x t}(y)\right) F(x, y, t) \\
& +\int_{\mathbb{R}^{3}}[V(|x-z|)-V(|y-z|)] F(z, z, t) d z F(x, y, t) \\
& -\int_{\mathbb{R}^{3}}[V(|x-z|)-V(|y-z|)] F(x, z, t) F(z, y, t) d z
\end{aligned}
$$

in atomic units, where $V_{e x t}$ is the external potential energy and $V(r)=1 / r$ is the Coulomb interaction potential. The the last term on the right-hand side of (1I) is the "exchange" term.

The Coulomb potential, however, is not amenable to the techniques of this article because it is unbounded (this case being dealt with in [5). In this article, we consider interaction potentials given by a bounded function $V(|x-y|)$, or, more generally, any bounded, symmetric, two-body operator $V$ (not necessarily a multiplication operator). The one-particle energy operator will be denoted by $L$, the interaction energy operator for a single pair of particles will be denoted by $V$, and the total energy operator for a system of particles will be the sum of all single-particle energies and all pair energies. Although the number of particles does not change under the dynamics just described, we prefer to formulate the dynamics on a fermion Fock space so that we may consider initial states of indeterminate particle number. We are going to show that quasifree initial states enhance the accuracy of the TDHF approximation.

Let $L$ be a self-adjoint operator on a Hilbert space $\mathbb{H}$, and let $V$ be a bounded Hermitian operator on $\mathbb{H} \otimes \mathbb{H}$ that commutes with the transposition operator $U$ defined by $U(x \otimes y)=y \otimes x$. We are going to discuss the dynamics whose Hamiltonian $H$ on the fermion Fock space $\mathbb{F}_{\mathbb{H}}$ is written as

$$
H=\sum_{i, j}\langle j|L| i\rangle a_{j}^{\dagger} a_{i}+\sum_{i, j, k, l}\langle k l|V| i j\rangle a_{k}^{\dagger} a_{l}^{\dagger} a_{j} a_{i}
$$

in second quantized form. We will analyze the solutions of the von Neumann equation

$$
\begin{aligned}
i \frac{d}{d t} D(t) & =[H, D(t)] \\
D(0) & =D_{0},
\end{aligned}
$$

which is the evolution equation for the density operator on Fock space in the Schrödinger picture of quantum dynamics (in units of time and energy for which $\hbar=1$ ).

We will see that (31) leads to the following equation for the single-particle (number) density operator $\mathcal{N}_{1}(t)$ :

$$
\begin{aligned}
i \frac{d}{d t} \mathcal{N}_{1}(t) & =\left[L, \mathcal{N}_{1}(t)\right]+\left[V, \mathcal{N}_{2}(t)\right]_{: 1} \\
\mathcal{N}_{1}(0) & =\mathcal{N}_{1}\left(D_{0}\right)
\end{aligned}
$$


where $\left[V, \mathcal{N}_{2}(t)\right]_{: 1}$ denotes the partial trace of the two-particle operator $\left[V, \mathcal{N}_{2}(t)\right]$. Equation (41) for $\mathcal{N}_{1}(t)$ is not "closed" since its right hand side involves the two-particle density operator $\mathcal{N}_{2}(t)$. The TDHF approximation to $\mathcal{N}_{1}(t)$ is the solution of the initial value problem

$$
\begin{aligned}
i \frac{d}{d t} F(t) & =[L, F(t)]+\left[V, F(t)^{\otimes 2} 2 A_{2}\right]_{: 1} \\
F(0) & =\mathcal{N}_{1}(D(0))
\end{aligned}
$$

where $A_{2}$ is the orthogonal projector of $\mathbb{H} \otimes \mathbb{H}$ onto the subspace of antisymmetric vectors. The existence and uniqueness of solutions of (5) were established in [7] for the case where $V$ is a bounded operator, and in [10, 8] for the case where $V$ is a Coulombic interaction.

The TDHF equation (5) is obtained by closing the single-particle equation (4) with the Ansatz

$$
\mathcal{N}_{2}=\left(\mathcal{N}_{1} \otimes \mathcal{N}_{1}\right) 2 A_{2}
$$

at all times. The relation (6) holds for pure states corresponding to Slater determinants, and also for Gibbs densities. However, even supposing that $\mathcal{N}_{2}(0)$ satisfies (6), the interaction $V$ is likely to introduce "correlations" in $\mathcal{N}_{2}(t)$, that is, departures from (6), and ignoring those correlations in the TDHF equation requires justification.

We are going to prove that the absence of correlations is self-perpetuating in the mean field limit. Theorem 6.2 states that if $\mathcal{N}_{2}(0)$ satisfies (6) then $\mathcal{N}_{2}(t)$ asymptotically satisfies (6) as the number of particles $n$ tends to infinity and the interaction strength is scaled as $1 / n$. In this scaling, the force exerted on each individual particle by the $n-1$ other particles is of $O(1)$ as $n \rightarrow \infty$. This was called the "mean-field scaling" by H. Spohn in his fundamental review paper [14, where he derives the time-dependent Hartree equation. In the appendix we prove an important special case of Theorem 5.7 of [14] as a corollary of our Theorem 6.2.

Technically, we rely on the trace norm approach used in [14 to derive the Hartree equation. We first published our derivation of the TDHF equation in the mean field limit in 3 for initial states of fixed particle number such as Slater determinants. The main advance of this article is that the initial states need not be Slater determinants; the TDHF approximation should work equally well (or badly) for any quasifree initial state. Also, in this article we are not only interested in the mean field limit, and we derive the error bound of Theorem 6.1 for the unscaled problem.

Throughout this paper, $n$ denotes a fixed number of particles, $N$ denotes the the number operator on the Fock space over $\mathbb{H}$, and $\mathcal{N}_{1}$ denotes the number density operator on $\mathbb{H}$.

\section{Definitions and notation}

Consider a quantum particle whose Hilbert space is $\mathbb{H}$, i.e., a particle which, in isolation, would constitute a system whose (pure) quantum states are represented by the rank-one orthogonal 
projectors on some Hilbert space $\mathbb{H}$. The set of quantum states available to a system of $n$ particles of this kind is determined by their "statistics," i.e., whether the particles are fermions, bosons, or distinguishable. If the particles are fermions, a (pure) state of a system of $n$ of them is represented by a rank-one projectors onto vectors in the antisymmetric subspace $\mathbb{H}^{(n)}$ of the tensor power space $\mathbb{H}^{\otimes n}$. If the number of fermions in the system is not fixed, the appropriate Hilbert space is the fermion Fock space

$$
\mathbb{F}_{\mathbb{H}}=\mathbb{H}^{(0)} \oplus \mathbb{H}^{(1)} \oplus \mathbb{H}^{(2)} \oplus \mathbb{H}^{(3)} \oplus \cdots
$$

The possibility of a zero-particle state is accommodated by $\mathbb{H}^{(0)}$, a one-dimensional space spanned by the vacuum vector. We denote the number operator on $\mathbb{F}_{\mathbb{H}}$ by $N$.

Let $\Pi_{n}$ denote the group of permutations of $\{1,2, \ldots, n\}$. For each $\pi \in \Pi_{n}$, a unitary operator $U_{\pi}$ on $\mathbb{H}^{\otimes n}$ may be defined by extending

$$
U_{\pi}\left(x_{1} \otimes x_{2} \otimes \cdots \otimes x_{n}\right)=x_{\pi^{-1}(1)} \otimes x_{\pi^{-1}(2)} \otimes \cdots \otimes x_{\pi^{-1}(n)}
$$

to all of $\mathbb{H}^{\otimes n}$. The operator

$$
A_{n}=\frac{1}{n !} \sum_{\pi \in \Pi_{n}} \operatorname{sgn}(\pi) U_{\pi}
$$

is the orthogonal projector with range $\mathbb{H}^{(n)}$. If $x_{1}, x_{2}, \ldots, x_{n}$ is an orthonormal system in a Hilbert space $\mathbb{H}$, then the vector

$$
\sqrt{n !} A_{n}\left(x_{1} \otimes x_{2} \otimes \cdots \otimes x_{n}\right),
$$

is a unit vector in $\mathbb{H}^{(n)}$, called a Slater determinant.

We will consider many-particle states that are represented by density operators on $\mathbb{F}_{\mathbb{H}}$. We will only consider density operators that commute with $N$ and such that $N^{m} D$ is trace class for all $m \in \mathbb{N}$. For such densities $D$, one can define the reduced density operators $\mathcal{N}_{m}(D)$ of all orders $m$ :

If $T$ is a trace class operator on $\mathbb{H}^{(n)}$, and $m \leq n$, we will use the subscript notation $T_{: m}$ to denote the partial trace of $T$ of order $m$, a trace class operator on $\mathbb{H}^{(m)}$. (This operator is defined unambiguously thanks to the symmetry of $T$ considered as an operator on $\mathbb{H}^{\otimes n}$.) If $T$ is a density operator on $\mathbb{H}^{(n)}$, the operator $T_{: m}$ is known as the m-particle reduced density operator [13] and it is used to determine the expected values of the $m$-particle observables. Let $D$ be a density operator on $\mathbb{F}_{\mathbb{H}}$ that commutes with $N$. Then

$$
D=\bigoplus_{n=0}^{\infty} D_{n}
$$

where each $D_{n}$ is a nonnegative trace class operator on $\mathbb{H}^{(n)}$. Assuming that

$$
\operatorname{Tr}\left(N^{m} D\right)=\sum_{n=0}^{\infty} n^{m} \operatorname{Tr}\left(D_{n}\right)<\infty,
$$


we may define the $m^{\text {th }}$ order reduced density operator

$$
\mathcal{N}_{m}(D)=\sum_{n=m}^{\infty} \frac{n !}{(n-m) !} D_{n: m}
$$

where $D_{n: m}=\left(D_{n}\right)_{: m}$ is the partial trace of $D_{n}$. The reduced density $\mathcal{N}_{m}$ serves to describe the $m$-particle correlations in a system of many particles (see Section 6.3.3 of [9]).

\section{Dynamics and the BBGKY hierarchy}

We now define the dynamics (3) and the reduced dynamics (41).

Let $H$ be a self-adjoint operator on $\mathbb{H}$, and let $V$ be a bounded Hermitian operator on $\mathbb{H} \otimes \mathbb{H}$ that commutes with the transposition operator $U(x \otimes y)=y \otimes x$. For $1 \leq j \leq n$, let $L_{j}$ denote the operator

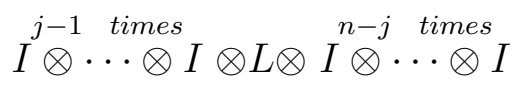

on $\mathbb{H}^{\otimes n}$ (the value of $n \geq j$ is not explicit in the notation $L_{j}$ but it will always be clear from context). For $1 \leq i<j \leq n$, let $U_{(i j)}$ denote the permutation operator on $\mathbb{H}^{\otimes n}$ that transposes the $i^{\text {th }}$ and $j^{\text {th }}$ factors of any simple tensor $x_{1} \otimes \cdots \otimes x_{n}$, and let

$$
V_{i j}=U_{(1 i)} U_{(2 j)}\left(V \otimes I^{\otimes n-2}\right) U_{(2 j)} U_{(1 i)}
$$

(again, the domain $\mathbb{H}^{\otimes n}$ of $V_{i j}$ will always be clear from context). For each $n$, define the operators

$$
\begin{aligned}
L^{(n)} & =\sum_{j=1}^{n} L_{j} \\
H^{(n)} & =L^{(n)}+\sum_{1 \leq i<j \leq n} V_{i j}
\end{aligned}
$$

on $\mathbb{H}^{(n)}$ (these operators are defined on all of $\mathbb{H}^{\otimes n}$ but we are only considering their restrictions to the invariant subspace $\left.\mathbb{H}^{(n)}\right)$. The Hamiltonian operator $H$, which we had formally represented above by (2), is the direct sum $H=\bigoplus H^{(n)}$ defined on the domain

$$
\mathcal{D}(H)=\left\{x=\oplus x_{n} \in \mathbb{F}_{\mathbb{H}}: \sum_{n}\left\|H^{(n)} x_{n}\right\|^{2}<\infty\right\} .
$$

This operator is closed and self-adjoint (see Section 6.3.1 of [9]) and $-i H$ is the generator of the strongly continuous group

$$
W_{t}=\bigoplus_{n=1}^{\infty} W_{t}^{(n)}
$$


of unitary operators on $\mathbb{F}_{\mathbb{H}}$, where $W_{t}^{(n)}=\exp \left(-i t H^{(n)}\right)$. The dynamics corresponding to (3) are given by the group

$$
\mathcal{W}_{t}(D)=W_{t} D W_{-t}
$$

of isometries of the space of Hermitian trace class operators on $\mathbb{F}_{\mathbb{H}}$. (See Proposition 3.4 of [7] for a proof that groups of isometries defined in this way are strongly continuous.) It is convenient to have some notation for the free part of the dynamics, so we define

$$
\mathcal{U}_{t}^{(n)}(T)=U_{t}^{(n)} T U_{-t}^{(n)}
$$

with $U_{t}^{(n)}=\exp \left(-i t L^{(n)}\right)$.

The dynamical equation for the $m^{\text {th }}$ order reduced density $\mathcal{N}_{m}$ can be derived from (13) if the density $D$ satisfies the moment condition (11). The details of the derivation are provided in [4].

Proposition 3.1 Let $\mathcal{U}_{t}$ and $\mathcal{W}_{t}$ be as defined in (14) and (13).

Suppose that $D$ is a density operator on $\mathbb{F}_{\mathbb{H}}$ of the form $D=\oplus D_{n}$ such that (11) holds for some $m \in \mathbb{N}$. Let $\mathcal{N}_{m}(t)$ denote $\mathcal{N}_{m}\left(\mathcal{W}_{t}(D)\right)$. Then $\mathcal{N}_{m}(t)$ satisfies

$\mathcal{N}_{m}(t)=\mathcal{U}_{t}^{(m)} \mathcal{N}_{m}(0)-i \int_{0}^{t} \mathcal{U}_{t-s}^{(m)} \sum_{1 \leq i<j \leq m}\left[V_{i j}, \mathcal{N}_{m}(s)\right] d s-i \int_{0}^{t} \mathcal{U}_{t-s}^{(m)} \sum_{j=1}^{m}\left[V_{j, m+1}, \mathcal{N}_{m+1}(s)\right]_{: m} d s$

These equations for the reduced density operators are known as the BBGKY hierarchy. The first equation of the hierarchy is equation (41) in integral form.

\section{The TDHF hierarchy}

The existence and uniqueness of mild solutions of the TDHF equation (15) is established in [7]. There it is shown that the integral equation

$$
F(t)=\mathcal{U}_{t}^{(1)} F(0)-i \int_{0}^{t} \mathcal{U}_{t-s}^{(1)}\left[V, F(s)^{\otimes 2} 2 A_{2}\right]_{: 1} d s
$$

has a unique solution $F(t)$ for any Hermitian trace class operator $F(0)$. Define $\mathcal{F}_{1}(t)=F(t)$ and, for $m>1$, define

$$
\mathcal{F}_{m}(t)=F(t)^{\otimes m} m ! A_{m}
$$

We proceed to derive equations for the $\mathcal{F}_{m}(t)$ from (15).

First, set $G(t)=\mathcal{U}_{-t}^{(1)} F(t)$, so that

$$
G(t)=F(0)-i \int_{0}^{t} \mathcal{U}_{-s}^{(1)}\left[V, F(s)^{\otimes 2} 2 A_{2}\right]_{: 1} d s .
$$


Now apply the product rule (in integral form) to $G(t)^{\otimes m}$ :

$$
\begin{aligned}
G(t)^{\otimes m} & =F(0)^{\otimes m}-i \sum_{j=1}^{m} \int_{0}^{t} G(s)^{\otimes j-1} \otimes \mathcal{U}_{-s}^{(1)}\left[V, F(s)^{\otimes 2} 2 A_{2}\right]_{: 1} \otimes G(s)^{\otimes n-j} d s \\
& =F(0)^{\otimes m}-i \int_{0}^{t} \mathcal{U}_{-s}^{(m)} \sum_{j=1}^{m}\left[V_{j, m+1}, F(s)^{\otimes m+1}\left(I-U_{(j, m+1)}\right)\right]_{: m} d s .
\end{aligned}
$$

Apply $\mathcal{U}_{t}^{(m)}$ to both sides of the preceding equation to obtain

$$
F(t)^{\otimes m}=\mathcal{U}_{t}^{(m)} F(0)^{\otimes m}-i \int_{0}^{t} \mathcal{U}_{t-s}^{(m)} \sum_{j=1}^{m}\left[V_{j, m+1}, F(s)^{\otimes m+1}\left(I-U_{(j, m+1)}\right)\right]_{: m} d s .
$$

Multiply both sides of the last equation by $m ! A_{m}$ on the left, noting that $\mathcal{U}_{s}^{(m)}(X) A_{m}=$ $\mathcal{U}_{s}^{(m)}\left(X A_{m}\right)$ and that $A_{m}$ commutes with $\sum V_{j, m+1}$ :

$$
\mathcal{F}_{m}(t)=\mathcal{U}_{t}^{(m)} \mathcal{F}_{m}(0)-i \int_{0}^{t} \mathcal{U}_{t-s}^{(m)} \sum_{j=1}^{m}\left[V_{j, m+1}, F(s)^{\otimes m+1}\left(I-U_{(j, m+1)}\right) m ! A_{m}\right]_{: m} d s .
$$

Since $(m+1) ! A_{m+1}=\left(I-U_{(1, m+1)} \cdots-U_{(m, m+1)}\right) m ! A_{m}$, the last equation may be rewritten

$$
\begin{aligned}
\mathcal{F}_{m}(t) & =\mathcal{U}_{t}^{(m)} \mathcal{F}_{m}(0)-i \int_{0}^{t} \mathcal{U}_{t-s}^{(m)} \sum_{j=1}^{m}\left[V_{j, m+1}, \mathcal{F}_{m+1}(s)\right]_{: m} d s \\
& +\sum_{1 \leq j \neq k \leq m} i \int_{0}^{t} \mathcal{U}_{t-s}^{(m)}\left[V_{j, m+1}, F(s)^{\otimes m+1} U_{(k, m+1)} m ! A_{m}\right]_{: m} d s
\end{aligned}
$$

We call these equations for the $\mathcal{F}_{m}(t)$ the TDHF hierarchy.

\section{Estimates}

In this section we have collected some estimates used in the proofs of Theorems6.1 and 6.2. The first two of the following propositions are stated here without proof, for the facts are known, and the reader may find proofs of them in [4]. We use the notation $\|\cdot\|$ for the operator norm and $\|\cdot\|_{1}$ for the trace norm.

Proposition 5.1 If $D$ is a density operator on $\mathbb{F}_{\mathbb{H}}$ that commutes with $N$ and such that $\operatorname{Tr}(N D)<\infty$, then the operator norm of $\mathcal{N}_{1}(D)$ is not greater than 1 . 
Proposition 5.2 If $T$ is a Hermitian trace class operator then

$$
\left\|T^{\otimes n} n ! A_{n}\right\|_{1} \leq\|T\|_{1}^{n} .
$$

Proposition 5.3 The trace norm of the last term in (17) does not exceed

$$
2 m(m-1)\|V\|\|F(0)\|\|F(0)\|_{1}^{m} t .
$$

Proof: $\quad$ The trace norm of the last term in (17) is bounded by

$$
2 m(m-1) \int_{0}^{t}\left\|\left\{V_{m-1, m+1} U_{(m, m+1)}\left(\mathcal{F}_{m}(s) \otimes F(s)\right)\right\}_{: m}\right\|_{1} d s
$$

thanks to the symmetry of $\mathcal{F}_{m}$. It can be verified that

$$
\left(V_{m-1, m+1} U_{(m, m+1)}\left(\mathcal{F}_{m}(s) \otimes F(s)\right)\right)_{: m}=\left(I^{\otimes m-1} \otimes F(s)\right) V_{m-1, m} \mathcal{F}_{m}(s),
$$

whence (19) is bounded by

$$
2 m(m-1) \int_{0}^{t}\|V\|\|F(s)\|\left\|\mathcal{F}_{m}(s)\right\|_{1} d s .
$$

Now $\left\|\mathcal{F}_{m}(s)\right\|_{1} \leq\|F(s)\|_{1}^{m}$ by Proposition 5.2 since $\mathcal{F}_{m}=F^{\otimes m} m ! A_{m}$. Furthermore, $\|F(s)\|_{1}=$ $\|F(0)\|_{1}$ and $\|F(s)\|=\|F(0)\|$ for all $s>0$ by Proposition 4.3 of [7]. Thus we arrive at the bound stated in the Proposition.

\section{Accuracy of the TDHF approximation}

In this section we will compare the single-particle density operator $\mathcal{N}_{1}(t)$ to its approximation by the solution of the TDHF equation

$$
\begin{aligned}
& F(t)=\mathcal{U}_{t}^{(1)} F(0)-i \int_{0}^{t} \mathcal{U}_{t-s}^{(1)}\left[V, F(s)^{\otimes 2} 2 A_{2}\right]_{: 1} d s \\
& F(0)=\mathcal{N}_{1}(0) .
\end{aligned}
$$

We can control the distance between $\mathcal{N}_{1}(t)$ and $F(t)$ in trace norm when the initial manyparticle state is gauge-invariant quasifree. Theorems 6.1 and 6.2 show how the accuracy of the TDHF approximation is enhanced when the initial state involves many uncorrelated fermions.

We consider the class of initial states that have density operators $D$ of the form (10) such that $\operatorname{Tr}(N D)<\infty$ and

$$
\mathcal{N}_{n}(D)=\mathcal{N}_{1}(D)^{\otimes n} n ! A_{n}
$$


for all $n \in \mathbb{N}$. These relations characterize the gauge-invariant quasifree states of the CAR algebra having a trace class single-particle reduced density operator $\mathcal{N}_{1} 9$.

There are two important examples of such initial states: Slater densities and Gibbs grand canonical equilibrium states. "Slater densities" are those of the form

$$
D=\mathbf{0} \oplus \cdots \oplus \text { times }^{n-1}(|\psi\rangle\langle\psi|) \oplus \mathbf{0} \oplus \cdots,
$$

where $\psi$ is an $n$-particle Slater determinant (91). Gibbs equilibrium states are obtained as follows: let $H$ be the single-particle Hamiltonian and $d \Gamma(H)$ its functorial extension to the fermion Fock space viewed as the exterior algebra of $\mathbb{H}$. In other words,

$$
d \Gamma(H)=\bigoplus_{n \geq 0} d \Gamma_{n}(H)
$$

where $d \Gamma_{n}(H)$ is the restriction to $\mathbb{H}^{(n)}$ of the operator

$$
\sum_{j=1}^{n} I \otimes \ldots \otimes \text { times } \stackrel{n-j \text { times }}{i-\ldots} I \otimes H \otimes I \otimes \ldots \otimes I
$$

Whenever $\beta>0$ is such that $e^{-\beta H}$ is trace-class on $\mathbb{H}$, the Gibbs equilibrium state at inverse temperature $\beta$ with chemical potential $\mu$ is defined by the density operator proportional to $\exp (-\beta d \Gamma(H-\mu I))$ (see Proposition 5.2.22 of [9] for more details on Gibbs states).

Theorem 6.1 Let $D$ be the density operator on $\mathbb{F}_{\mathbb{H}}$ of a gauge-invariant quasifree state with finite expected particle number, i.e., with $\operatorname{Tr}(N D)<\infty$. Let $\mathcal{N}_{1}(t)$ denote $\mathcal{N}_{1}\left(\mathcal{W}_{t}(D)\right)$, where $\mathcal{W}_{t}$ is the dynamics with two-particle interactions defined in (13).

Let $F(t)$ be the solution of the TDHF equation (20).

Let $\tau$ denote $\left(2\|V\|\left\|\mathcal{N}_{1}\right\|_{1}\right)^{-1}$. Then

$$
\left\|\mathcal{N}_{1}(t)-F(t)\right\|_{1} \leq \frac{3}{2}\left(\frac{t}{\tau-t}\right)^{2}
$$

for $t<\tau$.

The proof of this theorem is postponed until the end of this Section.

Unfortunately, the bound (22) on the error of the TDHF approximation is valid only when $t<\tau$ and we have no explicit bounds for larger $t$. In principle, the estimate of Theorem 6.1] could be used to establish that any effect observed in a TDHF simulation before the critical time $\tau$ reflects a true effect of the interaction. However, in the numerical tests we have conducted so far, very little appears to happen before the critical time $\tau$, and we fear that the estimate of 
Theorem 6.1 might not prove generally useful. Nonetheless, Theorem 6.1 does show that the error of the TDHF approximation is less than one might expect when the initial condition is an uncorrelated many-fermion state, for even at short times $t<\tau$ the left-hand side of (22) is proportional to $\|F(0)\|_{1}=\left\|\mathcal{N}_{1}(D(0))\right\|_{1}$ prima facie, not bounded independently of $\left\|\mathcal{N}_{1}\right\|_{1}$.

The improvement in accuracy of the TDHF approximation when the initial state is uncorrelated is even more evident in the "mean field scaling." It is in this spirit that we are about to introduce a coupling constant $\lambda$ into the interaction term of the many-particle system and consider a scalings where $\lambda$ times the average particle number tends to 0 or remains bounded. We do not discuss the physical significance of such scalings; we consider them only so that we may more easily express how the accuracy of the TDHF equation is affected by uncorrelated initial data.

For each value of the parameter $\lambda>0$, consider the Hamiltonian

$$
H_{\lambda}=\sum_{i, j}\langle j|L| i\rangle a_{j}^{\dagger} a_{i}+\lambda \sum_{i, j, k, l}\langle k l|V| i j\rangle a_{k}^{\dagger} a_{l}^{\dagger} a_{j} a_{i} .
$$

If the initial density operator represents a gauge-invariant quasifree state with finite expected particle number, then Proposition 3.1 implies that all reduced number density operators exist and satisfy

$$
\begin{aligned}
& \mathcal{N}_{\lambda m}(t)=\mathcal{U}_{t}^{(m)} \mathcal{N}_{\lambda m}(0)-\lambda i \int_{0}^{t} \mathcal{U}_{t-s}^{(m)}\left(\sum_{1 \leq i<j \leq m}\left[V_{i j}, \mathcal{N}_{\lambda m}(s)\right]+\sum_{j=1}^{m}\left[V_{j, m+1},\left(\mathcal{N}_{\lambda}\right)_{m+1}(s)\right]_{: m}\right) d s \\
& \mathcal{N}_{\lambda m}(0)=\mathcal{N}_{m}\left(D_{\lambda}(0)\right)
\end{aligned}
$$

The TDHF equation corresponding to (23) is

$$
\begin{aligned}
& F_{\lambda}(t)=\mathcal{U}_{t}^{(1)} F_{\lambda}(0)-\lambda i \int_{0}^{t} \mathcal{U}_{t-s}^{(1)}\left[V, F_{\lambda}(s)^{\otimes 2} 2 A_{2}\right]_{: 1} d s \\
& F_{\lambda}(0)=\mathcal{N}_{\lambda 1}(0) .
\end{aligned}
$$

Theorem 6.2 Let $\left\{D_{\lambda}\right\}_{\lambda>0}$ be a family of density operators on $\mathbb{F}_{\mathbb{H}}$ that represent gauge-invariant quasifree states with finite expected particle number. Let $\mathcal{N}_{\lambda m}(t)$ be the solution of (24) with initial condition $N_{\lambda m}(0)=N_{m}\left(D_{\lambda}\right)$ and let $F_{\lambda}(t)$ be the solution of the TDHF equation (25). Let $\mathcal{F}_{\lambda m}(t)=F_{\lambda}(t)^{\otimes m} m ! A_{m}$.

1) If $\lim _{\lambda \rightarrow 0} \lambda\left\|\mathcal{N}_{\lambda 1}(0)\right\|_{1}=0$ then, for each fixed $t>0$ and $m \in \mathbb{N}$,

$$
\left\|\mathcal{N}_{\lambda m}(t)-\mathcal{F}_{\lambda m}(t)\right\|_{1} /\left\|\mathcal{N}_{\lambda 1}\right\|_{1}^{m}=O(\lambda)
$$




$$
\begin{aligned}
& \text { If } \limsup _{\lambda \rightarrow 0} \lambda\left\|\mathcal{N}_{\lambda 1}(0)\right\|_{1}<\infty \text { then } \\
& \qquad \lim _{\lambda \rightarrow 0}\left\|\mathcal{N}_{\lambda m}(t)-\mathcal{F}_{\lambda m}(t)\right\|_{1} /\left\|\mathcal{N}_{\lambda 1}\right\|_{1}^{m}=0
\end{aligned}
$$

for all $t>0$ and all $m \in \mathbb{N}$.

\section{Remark on the persistence of the interaction in the mean field limit}

Although we have assumed only that the interaction $V$ is a bounded and symmetric twoparticle operator, we are mainly interested in the case where it is a bounded multiplication operator. In this case one may bound the exchange term in the TDHF equation as follows. The exchange term in (11) is the sum of two products: the product of the integral operators with kernels $V(|x-z|) F(x, z)$ and $F(z, y)$, and the product of the integral operators with kernels $F(x, z)$ and $V(|y-z|) F(z, y)$. The trace norm of each of these products is bounded by the product of the Hilbert-Schmidt norms of its factors. Since the operator norm of a fermionic single-particle operator is less than or equal to 1 (viz. Proposition [5.1)

$$
\|F\|_{H S}=\sqrt{\operatorname{Tr}\left(F^{2}\right)} \leq \sqrt{\|F\|_{1}\|F\|} \leq \sqrt{\|F\|_{1}}
$$

and it follows that the trace norm of the exchange term is bounded by $2\|F\|_{1}\|V\|_{L^{\infty}}$. Thus the contribution of the exchange term is not much larger than the error of the TDHF approximation itself! To see this more clearly, let us return to Statement 1 of Theorem 6.2. Squeezing the most we can out of its proof informs us that

$$
\left\|\mathcal{N}_{\lambda 1}(t)-F_{\lambda}(t)\right\|_{1}=O\left(\lambda^{2}\left\|F_{\lambda}\right\|_{1}^{2}\right)
$$

if $\lim _{\lambda \rightarrow 0} \lambda\left\|F_{\lambda}\right\|_{1}=0$. (It is due to the fact that the $j=0$ term of the second series on the right-hand side of the bound in Lemma 6.1 below vanishes when $m=1$.) But the part of $F_{\lambda}(t)$ due to the exchange term is $O\left(\lambda\left\|F_{\lambda}\right\|_{1}\right)$ by the above estimate, and $\lambda\left\|F_{\lambda}\right\|_{1}$ already tends to zero.

Knowing that the exchange effect vanishes in the mean field limit, one might wonder whether Theorem 6.2 is trivial. The theorem states that the error between the true single-particle density and its TDHF approximation tends to zero under certain conditions - but perhaps this so simply because the effect of the interaction disappears in the limits we have taken? At least the "direct" part of the interaction does not disappear in the limit, for it survives as the nonlinear term in the time-dependent Hartree equation as shown in the following corollary of Theorem 6.2

Corollary 6.1 Let $\psi \in L^{2}\left(\mathbb{R}^{3}\right)$ have norm one, and for $n \in \mathbb{N}$ let $\Psi_{n}(x, t) \in L^{2}\left(\mathbb{R}^{3 n}\right)$ be the solution of the Schrödinger equation

$$
\begin{aligned}
i \frac{\partial}{\partial t} \Psi_{n}(x, t) & =-\frac{1}{2} \sum_{j=1}^{n} \Delta_{x_{j}} \Psi_{n}(x, t)+\frac{1}{n} \sum_{i<j} V\left(\left|x_{i}-x_{j}\right|\right) \Psi_{n}(x, t) \\
\Psi_{n}(x, 0) & =\psi\left(x_{1}\right) \psi\left(x_{2}\right) \cdots \psi\left(x_{n}\right)
\end{aligned}
$$


with $V(x)$ bounded. Let $\rho_{n}(x, y, t)$ denote the integral kernel of the single-particle density operator, normalized to have trace 1 rather than $n$ :

$$
\rho_{n}(x, y, t)=\int_{\mathbb{R}^{3}} \cdots \int_{\mathbb{R}^{3}} \overline{\Psi_{n}\left(y, z_{2}, \ldots, z_{n}, t\right)} \Psi_{n}\left(x, z_{2}, \ldots, z_{n}, t\right) d z_{2} \cdots d z_{n} .
$$

Let $\rho_{*}(x, y, t)$ denote the solution of the time-dependent Hartree equation

$$
\begin{aligned}
i \frac{\partial}{\partial t} \rho_{*}(x, y, t) & =-\frac{1}{2}\left(\Delta_{x}-\Delta_{y}\right) \rho_{*}(x, y, t)+\int_{\mathbb{R}^{3}}[V(|x-z|)-V(|y-z|)] \rho_{*}(z, z, t) d z \rho_{*}(x, y, t) \\
\rho_{*}(x, y, 0) & =\overline{\psi(y)} \psi(x) .
\end{aligned}
$$

Then $\rho_{n}(t)$ converges in trace norm to $\rho_{*}(t)$ at each fixed $t \geq 0$ as $n \longrightarrow \infty$.

The proof of this corollary is given in the appendix.

\section{Proof of Theorems 6.1 and 6.2}

Let $\mathcal{N}_{m}(t)$ be as in Proposition [3.1, and let $\mathcal{F}_{m}(t)$ satisfy the TDHF hierarchy. In the hypotheses Theorems 6.1 and 6.2 we suppose that $F(0)=\mathcal{N}_{1}(D(0)$ ), but for now let us only assume that

$$
\|F(0)\| \leq 1 \quad \text { and } \quad\|F(0)\|_{1}=\left\|\mathcal{N}_{1}(D(0))\right\|_{1} .
$$

The trace norm of $\mathcal{N}_{1}(t)$ is independent of $t$, and we shall denote it simply by $\left\|\mathcal{N}_{1}\right\|_{1}$. Assuming (29), the bound of Proposition 5.3 is itself bounded by

$$
m(m-1) 2\|V\|\left\|\mathcal{N}_{1}\right\|_{1}^{m} t .
$$

Subtracting equations (17) from the BBGKY equations of Proposition 3.1] and using (30) leads to the estimates

$$
\begin{aligned}
\left\|\mathcal{N}_{m}(t)-\mathcal{F}_{m}(t)\right\|_{1} & \leq\left\|\mathcal{N}_{m}(0)-\mathcal{F}_{m}(0)\right\|_{1}+m(m-1)\|V\|\left(2\left\|\mathcal{N}_{1}\right\|_{1}^{m}+\left\|\mathcal{N}_{m}\right\|_{1}\right) t \\
& +m 2\|V\| \int_{0}^{t}\left\|\mathcal{N}_{m+1}(s)-\mathcal{F}_{m+1}(s)\right\|_{1} d s
\end{aligned}
$$

Iterating this estimate $k$ times, one obtains

$$
\begin{aligned}
\left\|\mathcal{N}_{m}(t)-\mathcal{F}_{m}(t)\right\|_{1} & \leq \sum_{j=0}^{k} a_{m+j}\left(\begin{array}{c}
m+j-1 \\
j
\end{array}\right) C^{j} t^{j}+\sum_{j=0}^{k} \frac{b_{m+j}}{j+1}\left(\begin{array}{c}
m+j-1 \\
j
\end{array}\right) C^{j} t^{j+1} \\
& +C^{n} \frac{(m+k) !}{(m-1) !} \int_{0}^{t} \int_{0}^{t_{1}} \cdots \int_{0}^{t_{n}}\left\|\mathcal{N}_{m+k+1}(s)-\mathcal{F}_{m+k+1}(s)\right\|_{1} d s d t_{k} \cdots d t_{1}
\end{aligned}
$$


with $C=2\|V\|$ and

$$
\begin{aligned}
& a_{m}=\left\|\mathcal{N}_{m}(0)-\mathcal{F}_{m}(0)\right\|_{1} \\
& b_{m}=m(m-1)\|V\|\left(2\left\|\mathcal{N}_{1}\right\|_{1}^{m}+\left\|\mathcal{N}_{m}\right\|_{1}\right) .
\end{aligned}
$$

To make use of these estimates we need some control over the size of the integrand in (31). We will assume that

$$
\left\|\mathcal{N}_{m}\right\|_{1} \leq\left\|\mathcal{N}_{1}\right\|_{1}^{m}
$$

for all $m$, for this bound holds for gauge-invariant quasifree state with finite expected particle number by (21) and Proposition 5.2. Note that (33) holds independently of time, since the dynamics conserve particle number. With the bound (33), the last term on the right hand side of (31) may be bounded by

$$
2\left(\begin{array}{c}
m+k \\
m-1
\end{array}\right)\left(C\left\|\mathcal{N}_{1}\right\|_{1} t\right)^{m+k+1}
$$

which tends to 0 as $k$ tends to infinity if $m$ fixed and $C\left\|\mathcal{N}_{1}\right\|_{1} t<1$. Furthermore, assuming (33), we can bound $b_{m}$ of (32) by $\frac{3}{2} C\left\|\mathcal{N}_{1}\right\|_{1}^{m} m(m-1)$ and establish the following lemma:

Lemma 6.1 Suppose that $D$ is a density operator on $\mathbb{F}_{\mathbb{H}}$ of the form $D=\oplus D_{n}$, such that (33) holds for all $m \in \mathbb{N}$. Let $\mathcal{W}_{t}$ be as defined in (13) and let $\mathcal{N}_{m}(t)$ denote $\mathcal{N}_{m}\left(\mathcal{W}_{t}(D)\right)$. Let $F(t)$ be the solution of a TDHF equation (15) whose initial condition $F(0)$ satisfies (29), and let $\mathcal{F}_{m}(t)$ be as in (16). Then, with $C=2\|V\|$,

$$
\begin{aligned}
\frac{\left\|\mathcal{N}_{m}(t)-\mathcal{F}_{m}(t)\right\|_{1}}{\left\|\mathcal{N}_{1}\right\|_{1}^{m}} & \leq \sum_{j=0}^{\infty} \frac{\left\|\mathcal{N}_{m+j}(0)-\mathcal{F}_{m+j}(0)\right\|_{1}}{\left\|\mathcal{N}_{1}\right\|_{1}^{m+j}}\left(\begin{array}{c}
m+j-1 \\
m-1
\end{array}\right)\left(C\left\|\mathcal{N}_{1}\right\|_{1} t\right)^{j} \\
& +\left\|\mathcal{N}_{1}\right\|_{1}^{-1} \frac{3}{2} \sum_{j=0}^{\infty}(m+j-1)\left(\begin{array}{c}
m+j \\
m-1
\end{array}\right)\left(C\left\|\mathcal{N}_{1}\right\|_{1} t\right)^{j+1}
\end{aligned}
$$

when $C\left\|\mathcal{N}_{1}\right\|_{1} t<1$.

In the hypotheses of Theorems 6.1 and 6.2 the initial data for the exact dynamics are assumed to be gauge-invariant quasifree states $D$ (or $D_{\lambda}$ ) with finite expected particle number, and the initial data for the corresponding TDHF equations are assumed to be $F(0)=\mathcal{N}_{1}(D)$ (or $F_{\lambda}(0)=\mathcal{N}_{1}\left(D_{\lambda}\right)$ ). Thus, the requirements (33) and (29) in Lemma 6.1 are satisfied under the hypotheses of Theorems 6.1 and 6.2 .

Theorem 6.1 follows from Lemma 6.1 since $\mathcal{N}_{m}(0)=\mathcal{F}_{m}(0)$ for all $m$ by (21).

To prove Theorem 6.2 we apply Lemma 6.1 to the many-particle system (13) and the TDHF equation (15) with $\lambda V$ in place of $V$. 
Statement 1 of the theorem comes easily: $\mathcal{N}_{\lambda m}(0)=\mathcal{F}_{\lambda m}(0)$ for all $m$, and Lemma 6.1 implies that

$$
\frac{\left\|\mathcal{N}_{\lambda m}(t)-\mathcal{F}_{\lambda m}(t)\right\|_{1}}{\left\|\mathcal{N}_{\lambda m}\right\|_{1}^{m}}<\lambda C t \frac{3}{2} \sum_{j=0}^{\infty} \frac{(m+j)^{m}}{(m-1) !}\left(\lambda C\left\|\mathcal{N}_{\lambda 1}\right\|_{1} t\right)^{j}
$$

with $C=2\|V\|$ when $\lambda C\left\|\mathcal{N}_{\lambda 1}\right\|_{1} t<1$. Statement 1 follows since $\lim _{\lambda \rightarrow 0} \lambda\left\|\mathcal{N}_{\lambda 1}\right\|_{1}=0$.

The proof of Statement 2 requires the fuller version of the inequality in Lemma 6.1. Since (33) and (29) are satisfied at any time $s>0$ if they are satisfied initially, Lemma 6.1 implies that

$$
\begin{aligned}
\frac{\left\|\mathcal{N}_{\lambda m}(s+\Delta t)-\mathcal{F}_{\lambda m}(s+\Delta t)\right\|_{1}}{\left\|\mathcal{N}_{\lambda 1}\right\|_{1}^{m}} & \leq \sum_{j=0}^{\infty} \frac{\left\|\left(\mathcal{N}_{\lambda}\right)_{m+j}(s)-\left(\mathcal{F}_{\lambda}\right)_{m+j}(s)\right\|_{1}}{\left\|\mathcal{N}_{\lambda 1}\right\|_{1}^{m+j}}\left(\begin{array}{c}
m+j-1 \\
m-1
\end{array}\right)\left(\lambda C\left\|\mathcal{N}_{\lambda 1}\right\|_{1} \Delta t\right)^{j} \\
& +\lambda C \Delta t \frac{3}{2} \sum_{j=0}^{\infty}(m+j-1)\left(\begin{array}{c}
m+j \\
m-1
\end{array}\right)\left(\lambda C\left\|\mathcal{N}_{\lambda 1}\right\|_{1} \Delta t\right)^{j}
\end{aligned}
$$

for any $s \geq 0$ as long as $\Delta t<\left(\lambda C\left\|\mathcal{N}_{\lambda 1}\right\|_{1}\right)^{-1}$. Since $u=C \limsup _{\lambda \rightarrow 0} \lambda\left\|\mathcal{N}_{\lambda 1}\right\|_{1}$ is finite by hypothesis, taking the lim sup of both sides of (34) shows that

$$
\lim _{\lambda \rightarrow 0}\left\|\mathcal{N}_{\lambda m}(t)-\mathcal{F}_{\lambda m}(t)\right\|_{1} /\left\|\mathcal{N}_{\lambda 1}\right\|_{1}^{m}=0 \quad \forall m \in \mathbb{N}
$$

holds at time $t=s+\Delta t$ if it holds at time $t=s$ and $\Delta t<1 / u$. Since (35) holds at $t=0$, an inductive argument proves that it holds at all times $t>0$.

\section{Appendix: the time-dependent Hartree equation}

Here we prove Corollary 6.1]

The initial condition of (26) is not available to $n$ fermions in $\mathbb{R}^{3}$, for it is in extreme violation of the Pauli Exclusion Principle. To derive (28) from our theorem about fermions we will introduce an auxilliary space $\ell^{2}(\mathbb{N})$ to allow Pauli exclusion to hold while the spatial part of the $n$-particle wavefunction is permitted have the form $\psi\left(x_{1}\right) \psi\left(x_{2}\right) \cdots \psi\left(x_{n}\right)$. We are going to apply Statement 2 of Theorem 6.2 where the single-particle Hilbert space $\mathbb{H}=L^{2}\left(\mathbb{R}^{3}\right) \otimes \ell^{2}(\mathbb{N})$.

Let $e_{1}, e_{2}, \ldots$ be an orthonormal sequence in $\ell^{2}(\mathbb{N})$ and let $D_{1 / n}(0)$ be the orthogonal projector on $\mathbb{F}_{\mathbb{H}}$ whose range is the span of the $n$-particle Slater determinant

$$
A_{n}\left(\left(\psi \otimes e_{1}\right) \otimes\left(\psi \otimes e_{2}\right) \otimes \cdots \otimes\left(\psi \otimes e_{n}\right)\right) .
$$

It is helpful to rearrange factors and write

$$
D_{1 / n}(0)=R_{n}\left(P_{\psi}^{\otimes n} \otimes S_{n}\right) R_{n}^{*},
$$


where $P_{\psi \otimes n}$ denotes projection onto the span of $\psi^{\otimes n}$, and $S_{n}$ denotes the rank-one orthogonal projector on $\ell^{2}(\mathbb{N})^{\otimes n}$ whose image is the span of the Slater determinant formed from $e_{1}, \ldots, e_{n}$, and $R_{n}$ is the unitary transformation from $L^{2}\left(\mathbb{R}^{3}\right)^{\otimes n} \otimes \ell^{2}(\mathbb{N})^{\otimes n}$ to $\left(L^{2}\left(\mathbb{R}^{3}\right) \otimes \ell^{2}(\mathbb{N})\right)^{\otimes n}$ that rearranges the factors of any simple tensor thus:

$$
R_{n}\left(\left(f_{1} \otimes f_{2} \otimes \cdots \otimes f_{n}\right) \otimes\left(s_{1} \otimes s_{2} \otimes \cdots \otimes s_{n}\right)\right)=\left(f_{1} \otimes s_{1}\right) \otimes \cdots \otimes\left(f_{n} \otimes s_{n}\right) .
$$

If $\Psi_{n}(t)$ is the solution of (26) then $P_{\Psi_{n}(t)}$ - the projector whose range is the span of $\Psi_{n}(t)$ satisfies the von Neumann equation

$$
\begin{aligned}
i \frac{d}{d t} P_{\Psi_{n}(t)} & =-\frac{1}{2} \sum_{j=1}^{n}\left[\Delta_{x_{j}}, P_{\Psi_{n}(t)}\right]+\frac{1}{n} \sum_{i<j}\left[M_{V i j}, P_{\Psi_{n}(t)}\right] \\
P_{\Psi_{n}(t)} & =P_{\psi} \otimes n
\end{aligned}
$$

wherein $M_{V i j}$ denotes the multiplication operator $M_{V i j} \phi(x)=V\left(\left|x_{i}-x_{j}\right|\right) \phi(x)$. Thus

$$
\begin{aligned}
i \frac{d}{d t} R_{n}\left(P_{\Psi_{n}(t)} \otimes S_{n}\right) R_{n}^{*} & =-\frac{1}{2} \sum_{j=1}^{n} R_{n}\left(\left[\Delta_{x_{j}}, P_{\Psi_{n}(t)}\right] \otimes S_{n}\right) R_{n}^{*}+\frac{1}{n} \sum_{i<j} R_{n}\left(\left[M_{V i j}, P_{\Psi_{n}(t)}\right] \otimes S_{n}\right) R_{n}^{*} \\
R_{n}\left(P_{\Psi_{n}(0)} \otimes S_{n}\right) R_{n}^{*} & =D_{1 / n}(0) .
\end{aligned}
$$

Now we define

$$
D_{1 / n}(t)=R_{n}\left(P_{\Psi_{n}(t)} \otimes S_{n}\right) R_{n}^{*}
$$

for all $t \geq 0$, and we use the same notation to denote the extension of $D_{1 / n}(t)$ to a density operator defined on all of $\mathbb{F}_{\mathbb{H}}$. From (37) it may be seen that $D_{1 / n}(t)$ is the solution of the von Neumann equation

$$
\begin{aligned}
i \frac{d}{d t} D_{1 / n}(t) & =\left[H_{1 / n}, D_{1 / n}(t)\right] \\
D_{1 / n}(0) & =R_{n}\left(P_{\psi \otimes n} \otimes S_{n}\right) R_{n}^{*}
\end{aligned}
$$

where $H_{1 / n}$ is the Hamiltonian (23) on $\mathbb{F}_{\mathbb{H}}$ with $L=-\frac{1}{2} \Delta \otimes I_{\ell^{2}(\mathbb{N})}$ and $V$ denoting the multiplication operator

$$
(V \phi)(x, j, y, k)=V(|x-y|) \phi(x, j, y, k)
$$

on $\left(L^{2}\left(\mathbb{R}^{3}\right) \otimes \ell^{2}(\mathbb{N})\right)^{\otimes 2}$ — a slight abuse of notation.

Set $\lambda=1 / n$. According to Statement 2 of Theorem 6.2

$$
\lim _{\lambda \rightarrow 0}\left\|\mathcal{N}_{\lambda 1}(t)-F_{1 / n}(t)\right\|_{1} /\left\|\mathcal{N}_{\lambda 1}\right\|_{1}=0
$$

for all $t>0$, where $\mathcal{N}_{\lambda 1}(t)=\mathcal{N}_{1}\left(D_{1 / n}(t)\right)$ and $F_{1 / n}$ satisfies the TDHF equation (25). One may verify that the single-particle density operator $\mathcal{N}_{1}\left(D_{1 / n}(t)\right)$ equals $\rho_{n}(t) \otimes P_{n}$ where $P_{n}$ denotes 
the orthogonal projector onto the span of $\left\{e_{1}, \ldots, e_{n}\right\} \subset \ell^{2}(\mathbb{N})$ and $\rho_{n}(t)$ is as defined in (27). On the other hand, we claim that $F_{1 / n}(t)=\xi_{\lambda}(t) \otimes P_{n}$ where $\xi_{\lambda}(t)$ denotes the operator on $L^{2}\left(\mathbb{R}^{3}\right)$ whose integral kernel satisfies

$$
\begin{aligned}
i \frac{\partial}{\partial t} \xi_{\lambda}(x, y, t)= & -\frac{1}{2}\left(\Delta_{x}-\Delta_{y}\right) \xi_{\lambda}(x, y, t) \\
& +\int_{\mathbb{R}^{3}}(V(|x-z|)-V(|y-z|)) \xi_{\lambda}(z, z, t) d z \xi_{\lambda}(x, y, t) \\
& -\frac{1}{n} \int_{\mathbb{R}^{3}}(V(|x-z|)-V(|y-z|)) \xi_{\lambda}(x, z, t) \xi_{\lambda}(z, y, t) d z \\
\xi_{\lambda}(x, y, 0)= & \frac{\psi(y)}{\psi}(x)=\rho_{n}(x, y, 0) .
\end{aligned}
$$

Accepting this claim for now, and noting that $\left\|\mathcal{N}_{1}\left(D_{1 / n}(t)\right)\right\|_{1}$ is always equal to $n$, we find that

$$
\lim _{n \rightarrow \infty}\left\|\rho_{n}(t)-\xi_{\lambda}(t)\right\|_{1}=0
$$

for all $t>0$, since $\left\|\mathcal{N}_{\lambda 1}(t)-F_{1 / n}(t)\right\|_{1}=\left\|\left(\rho_{n}(t)-\xi_{\lambda}(t)\right) \otimes P_{n}\right\|_{1}=n\left\|\rho_{n}(t)-\xi_{\lambda}(t)\right\|_{1}$. Equation (38) is a small perturbation of (28) when $n$ is large, and we may verify that $\xi_{\lambda}(t)$ converges to $\rho_{*}(t)$ by applying Gronwall's inequality to

$$
\begin{aligned}
i \frac{d}{d t}\left(\rho_{*}-\xi_{\lambda}\right) & =\left[L, \rho_{*}-\xi_{\lambda}\right]+\left[V,\left(\rho_{*}-\xi_{\lambda}\right) \otimes \rho_{*}\right]_{: 1} \\
& +\left[V, \xi_{\lambda} \otimes\left(\rho_{*}-\xi_{\lambda}\right)\right]_{: 1}+\frac{1}{n}\left[V,\left(\xi_{\lambda} \otimes \xi_{\lambda}\right) U\right]_{: 1} .
\end{aligned}
$$

First, we get rid of the term $\left[L, \rho_{*}-\xi_{\lambda}\right]$ by passing to the "interaction picture" and rewriting (40) as an equation for $\exp (i t L)\left(\rho_{*}-\xi_{\lambda}\right) \exp (-i t L)$. Upon integrating and taking the trace norm one obtains

$$
\left\|\rho_{*}(t)-\xi_{\lambda}(t)\right\|_{1} \leq 4\|V\| \int_{0}^{t}\left\|\rho_{*}(s)-\xi_{\lambda}(s)\right\|_{1} d s+\frac{2}{n}\|V\| .
$$

Gronwall's inequality implies that $\left\|\xi_{\lambda}(t)-\rho_{*}(t)\right\|_{1}=O(1 / n)$ as $n \longrightarrow \infty$ for fixed $t$, which implies with (39) that $\left\|\rho_{n}(t)-\rho_{*}(t)\right\|_{1} \longrightarrow 0$, as asserted in the corollary.

Finally, we verify that $\xi_{\lambda}(t) \otimes P_{n}$ satisfies (25) when $\xi_{\lambda}(t)$ satisfies (38):

$$
\begin{aligned}
i \frac{d}{d t} F_{1 / n}= & i \frac{d}{d t} \xi_{\lambda} \otimes P_{n}=\left(\left[-\frac{1}{2} \Delta, \xi_{\lambda}\right]+\left[M_{V}, \xi_{\lambda}^{\otimes 2}\left(I-\frac{1}{n} U\right)\right]_{: 1}\right) \otimes P_{n} \\
= & {\left[L, F_{1 / n}\right]+\frac{1}{n} \operatorname{Trace}_{3,4}\left[V,\left(\xi_{\lambda} \otimes P_{n}\right)^{\otimes 2}\right] } \\
& -\frac{1}{n} \operatorname{Trace}_{3,4}\left[V,\left(\xi_{\lambda} \otimes P_{n}\right)^{\otimes 2} U_{(12)(34)}\right]
\end{aligned}
$$


where Trace $_{3,4}$ denotes the partial trace over the third and fourth factors of the tensor product, and $U_{(13)(24)}$ denotes the unitary operator on $\left(L^{2}(\mathbb{R}) \otimes \ell^{2}(\mathbb{N})\right)^{\otimes 2}$ that exchanges the first and third factors simple tensor products as it exchanges their second and fourth factors. The second term on the right-hand side of (41) is multiplied by $1 / n$ to compensate for the Trace $\left(P_{n}\right)=n$ due to the partial trace over the fourth factor. But (41) is the differential form of (25) for $\lambda=1 / n$.

Acknowledgement. This research was supported by by the European network HYKE (contract HPRN-CT-2002-00282), the French-Austrian "Amadeus" program (ÖAD 19/2003)) and by the Austrian START project "Nonlinear Schrödinger and quantum Boltzmann equations" of N.J.M. (contract Y-137-Tec). F.G. acknowledges support from the French IUF.

We also express our gratitude to Xavier Blanc, Eric Cancès and Claude Le Bris for numerous valuable discussions.

\section{References}

[1] R. Alicki and J. Messer. Nonlinear quantum dynamical semigroups for many-body open systems, Journal of Statistical Physics 32 no. 2 (1983) 299 - 312

[2] C. Bardos, F. Golse and N.J. Mauser, Weak coupling limit of the $N$-particle Schrödinger equation, Mathematical Analysis and Applications 7 no. 2 (2000) 275-293

[3] C. Bardos, F. Golse, A.D. Gottlieb and N.J. Mauser, Mean field dynamics of fermions and the time-dependent Hartree-Fock equation, Journal de Mathématiques Pures et Appliqueés 82 no. 6 (2003) $665-683$

[4] C. Bardos, F. Golse, A.D. Gottlieb and N.J. Mauser, Archived technical report: http://xxx.lanl.gov/abs/math-ph/0303070

[5] C. Bardos, F. Golse, A.D. Gottlieb and N.J. Mauser. Manuscript.

[6] C. Bardos, L. Erdös, F. Golse, N.J. Mauser and H.-T. Yau, Derivation of the SchrödingerPoisson equation from the quantum $N$-particle Coulomb problem, C. R. Acad. Sci., t 334 (6) Série I Math. (2002) 515-520

[7] A. Bove, G. Da Prato, and G. Fano, An existence proof for the Hartree-Fock time-dependent problem with bounded two-body interaction, Comm. Math. Phys. 37 (1974) 183-191

[8] A. Bove, G. Da Prato, and G. Fano, On the Hartree-Fock time-dependent problem, Comm. Math. Phys. 49 (1976) 25-33 
[9] O. Bratteli and D. W. Robinson, Operator Algebras and Quantum Statistical Mechanics 2, Springer-Verlag, 1997

[10] J. M. Chadam and R. T. Glassey, Global existence of solutions to the Cauchy problem for time-dependent Hartree equations, Journal of Mathematical Physics 16 no. 5 (1976) $1122-1130$

[11] P. Dirac, Note on exchange phenomena in the Thomas atom, Proc. Cambridge Philosophical Society 26 (1930) pp. 376 - 385

[12] M. J. Jamieson, Time-dependent Hartree-Fock theory for atoms, International Journal of Quantum Chemistry 4 (1971) 103-115

[13] D. ter Haar, On the density matrices used in Hartree-Fock calculations, Physica 26 (1960) $1041-1044$

[14] H. Spohn, Kinetic Equations from Hamiltonian Dynamics, Reviews of Modern Physics 53 (1980) $600-640$ 